derne assai utile la consultazione. Siamo grati all'illustre Autore di aver menzionato con distinzione la letteratura italiana sull'argomento e ci auguriamo che nessun genetista trascuri di utilizzare questo importante volume.

\section{L. $G$.}

GEDDA L.: Il concetto di «Genius familiaris» della malattia nello studio della prognosi. Minerva Medica - Anno XLVI, Vol. I, n. 30, pp. IO4I-IO49.

L'A. ritiene che per uno studio adeguato delle malattie ereditarie sia necessario considerare che esse sono caratterizzate da una dimensione che supera quelle che riguardano l'individuo ammalato (candidato). Cioè bisogna precisare in ogni caso il meccanismo di transmissione tenendo conto dell'eterogenia, della penetranza, della variabilità, ecc. Queste valutazioni vengono raccolte nel concetto clinico di "Genius familiaris 》 della malattia. A titolo di esempio l'A. illustra un albero genealogico caratterizzato da fistula auris congenita in una coppia $M Z$ e in un soggetto mononato, da oxicefalia nella medesima coppia e in tre soggetti mononati e da brachitelefalangia in quattro soggetti mononati.

GERHARDT KURT: Die Glockenbecherleute in Mittel-und Westdeutschland. Stuttgart, Schwazerbarlt'sche Verlagsbuchhandlung. I953.

L'A. stesso chiama questo suo libro una «monografia » dei "cultori del vaso campaniforme », una popolazione preistorica, che costruiva vasi campaniformi. Secondo la storia, questi vasi avrebbero contenuto droghe stupefacenti, che furono distribuite dai «marchant adventurers 》 (commercianti avventurieri) sotto il nome di «birra» fra gli «autoctoni» per sottometterli alla « aristocrazia guerriera ».

Gli scienziati poi hanno elaborato uno studio antropologico del tipo «cultori del vaso campaniforme " e l'A. descrive in questo libro gli scheletri e i crani del popolo dei «cultori di vasi campaniformi 》, che sono stati trovati nella Germania occidentale e centrale. Egli parla dei vari tipi di crani, della loro struttura razziale e con grande precisione illustra $\mathrm{i}$ componenti razziali della popolazione "cultori di vasi campaniformi 》.

Prima si credeva di poter parlare di una somatologia unica della popolazione dei «cultori di vasi campaniformi » invece oggigiorno si parla - secondo l'A. - di un tipo " cultore di vaso campaniforme 》 con la « testa ripida planoccipitale 》 che si trova più spesso di tutti gli altri tipi. Ma poi c'è il tipo con la « testa rotonda curvo-occipitale e la faccia larga » e diversi altri tipi di crani.

Nella seconda parte del libro l'A. si occupa dell'origine della popolazione «cultori del vaso cam- paniforme " e dice che si tratta piuttosto di un miscuglio di razze. Secondo l'A. la popolazione « dei cultori di vasi campaniformi » è gente di una terra lontana immigrata nel territorio della Germania di oggi che egli considera come stranieri di paese e stranieri di sangue.

Alla fine del libro l'A. attribuisce l'espansione dello «stile ceramico» a una certa popolazione, cioè alla sua migrazione ed è di opinione che la popolazione della «strana e impressionante cultura di vasi campaniformi » fa parte della gente di una certa razza con la sua migrazione.

GiRHARDT, KURT: Vom Reifungswandel der meuschlichen Physiognomie. AR. der Wiss. u. der Lit. Wiesbaden, I954.

L'autore parla in questo suo libro del mutamento della fisionomia umana nel periodo dalla prima infanzia fino all'età di 25 anni.

Questi studi si occupano prima di tutto del profilo della testa umana, cominciando dalla radice del naso fino all'inizio del collo e del contorno della faccia totale. : "en-face ». Il profilo fu diviso in 3 parti: 1) Dorso nasale. 2) Regione naso-labiale. 3) Labbro inferiore - regione del mento.

L'autore sottolinea l'importanza del metodo gemellare nel suo modo di confrontare i gruppi dei $\mathrm{MZ}$ e $\mathrm{DZ}$, per spiegare le relazioni genetiche. Nel libro si parla della concordanza della forma del profilo nei $M Z$ nel periodo dei IO-I5 anni di età. Gli studi dimostravano che in 29 coppie di $\mathrm{MZ}$ nel corso di IO-I5 anni il profilo mutava in 24 coppie e in 12 coppie di questi la concordanza è stata ristabilita. Invece in 14 coppie $\mathrm{DZ}$ mutavano I3 coppie e solo 2 coppie sono diventate nuovamente concordi.

Anche il " complesso caratteristico » della regione nasolabiale fu sottoposto alle ricerche genetiche gemellari. In 15 su 18 coppie infantili $\mathrm{MZ}$ la regione nasolabiale era completamente concordante, invece su 22 coppie di DZ era solo una coppia concordante. Le coppie $M Z$ adolescenti presentano circa $50 \%$ di concordanza, invece in 52 coppie di adolescenti $\mathrm{DZ}$ solo 3 coppie erano concordanti. La forma curvata del septum, la forma e posizione delle labbra superiori sono perfettamente concordanti nelle 18 coppie MZ. Solo in una coppia di $M Z$ era discordante la forma curvata del septum nasale e la forma della punta del naso, mentre sulle 22 coppie $\mathrm{DZ}$ questi caratteri sono discordanti in I3 coppie.

In seguito si tratta nel libro della frequenza di mutamento delle regioni del mento. Nuovamente è sottolineata la concordanza nei gemelli $\mathrm{MZ}$ e DZ. In 23 coppie MZ 22 coppie erano concordanti, invece fra $22 \mathrm{DZ}$ solo 2 coppie erano concordanti. 
Riguardo alla «forma totale della faccia 》 fra 26 coppie di MZ 25 coppie erano concordanti, ( età di I-12 anni).

L'autore divide il contorno della faccia in 3 categorie: I. largo. 2. stretto. 3. medio. Egli ha constatato, che riguardo il mutamento della forma, solo il $4 \%$ degli individui rimangono sempre uguali, il $96 \%$ cambiano la forma dei contorni della loro faccia. Trattanto anche qui della concordanza fra i MZ e DZ, l'autore dice di aver trovato, che la somiglianza fra le coppie MZ e DZ diminusce graduatamente avanzando l'età.

Concludendo, 1'autore dice che il processo dello sviluppo è uno straordinario complesso di formazioni dinamiche. La sua tesi può essere così riassunta: una forma iniziale può rimanere o può essere mutata, una forma arrivata può derivare da una medesima forma o da un'altra o da diverse altre forme.

\section{A. $M$.}

Heimann Hans: Die Scopolaminwirkung. Base! S. Karger, I952.

La scopolamina, com'è noto, è un alcaloide estraibile, insieme con molti altri, da specie vegetali della famiglia delle Solanacee, $e$, farmacologicamente, appartiene infatti al gruppo dell'atropina. Storicamente pare accertato che i primi ad usarne siano stati, verso la fine del secolo scorso, gli abitanti della Siberia, i quali, dalle foglie e dalle radici essiccate di "Hyosciamus niger », ricche assai della drogha, ricavavano una forte bevanda ad azione stupefacente. Ma, secondo Hatwich, l'uso della scopolamina sembra possa farsi risalire assai più indietro, in quanto è stato possibile identificarla tra le misture usate dagli Arabi fin da tempi antichissimi e citate nelle "Mille e una notte».

In tempi recenti fu introdotta in terapia per la cura del morbo di Parkinson e del Parkinsonismo postencefalitico, nel quale ultimo specialmente, a seguito della sua somministrazione, si era potuta riscontrare una buona riduzione deila rigidità muscolare, controllata con lo Sclerometro di Mangold.

Il suo impiego fu esteso al trattamento degli schizofrenici, in cui agisce come calmante. Ma, se viene somministrata a soggetti psichicamente normali, mostra un'azione, in certo senso, paradossa, in quanto provoca uno stato di forte eccitamento, ricco di allucinazioni. Espresso scopo dell'A., nell'intraprendere le interessanti ricerche, illustrate dalla pubblicazione che recensiamo, è stato appunto quello d'indagare a fondo e col corredo di moderni mezzi di ricerca, tra cui l'elettroencefalografo, l'azione della sostanza su soggetti sani, offertisi volontariamente.

Al soggetto in esame, in condizioni generali buone ed in stato di non affaticamento, fu somministrato sottocute un'unica dose di I mg. di scopolamina.
Mentre il soggetto si trovava disteso supino sul letto, da parte dello sperimentatore veniva invitato a pronunciare numeri di otto cifre, a contare all'indietro partendo da 100, a scandire parole (tedesche!) di parecchie sillabe, a ripetere frasi lettegli, a eseguire disegni, su temi semplici, ma obbligati.

Di particolare interesse ci sono sembrati i protocolli relativi alle esperienze, cui si è spontaneamente sottoposta una collega. Da questi l'A. ha potuto ricavare molteplici dati, che convalidando le ricerche precedenti, purtroppo non pubblicate, di Kappes, e che gli hanno permesso di rilevare come l'azione della scopolamina si attui in 3 fasi distinte: nella prima di esse si ha perdita di ogni attività volontaria, la quale consenta di mantenersi in contatto col mondo esterno, e prevalenza dei disturbi vegetativi (sete progressivamente intensa, disturbi dell'accomodazione, alterazioni del polso, nel senso di un aumerto della frequenza e dell'insorgenza di anomalie del ritmo);

nella seconda, l'A. ha riscontrato una completa dissociazione psichica, resa più evidente dalla mancanza di ogni coerenza logica nel ragionamento, dalla perdita dell'orientamento, sia riferito al tempo, che allo spazio, da una frammentarietà di coscienza, che impedisce al soggetto di fissare comunque la propria attenzione. Questa fase, per diversi punti, può paragonarsi alla Sindrome di Korsakow, nell'« allucinosi alcoolica », da cui peraltro si distingue per la continua mutevolezza dei giudizi intenzionali. Il sensorio appare obnubilato e circoscritto, mentre l'ideazione risulta simile alle « isole d'un arcipelago ", che, pur essendo distaccate, devono in qualche modo esser congiunte sotto lo specchio dell'acqua;

la terza è quella della ripresa, in cui però si manifesta quasi totale amnesia per gli eventi occorsi durante la seconda fase.

Da queste esperienze l'A. ritrae l'opinione che l'azione della scopolamina non debba interessare direttamente il nucleo stesso della personalità, ma svolgersi più perifericamente, in maniera di coinvolgere soltanto le sue manifestazioni esteriori.

L'esame elettroencefalografico, praticato a intervalli regolari, rileva la presenza di alterazioni nella sfera intenzionale, tradotte graficamente da una generale riduzione delle onde alfa e dall'assoluta mancanza di un ritmo, ciò che, con grande verosimiglianza, depone per un interessamento diffuso dell'encefalo in toto.

W. ZELLER. Konstitution und Entwicklung. Verlag Psychologische Rundschau, Göttingen. 1952.

I rapporti tra "costituzione 》 e "sviluppo " sono qui presentati al lettore sotto un angolo visuale che si distacca alquanto dal solito, seguendo l'A. un suo criterio logico particolare che ce li pone l'una in funzione dell'altro ed entrambi in funzione 